\title{
Topology and Damage Tolerance Optimisation of an Island-Hopping Aircraft MLG Fitting using Generative Design
}

\author{
M. R. Mahalik ${ }^{1}$, A. Jusuf ${ }^{2}$, T. Dirgantara ${ }^{3}$, L. Gunawan ${ }^{4}$ \\ ${ }^{1}$ Aerospace Engineering Study Program, Undergraduate Student \\ ${ }^{2}$ Lightweight Structures Research Group \\ Faculty of Mechanical and Aerospace Engineering \\ Institut Teknologi Bandung, Bandung, Indonesia
}

\begin{abstract}
In this paper, a topology and damage tolerance design optimisation of the Main Landing Gear (MLG) fitting of a 19 passengers' turboprop island-hopping aircraft was conducted by utilising the Generative Design technique. Generative Design technique has revolutionised the method of designing a structural component to attain the optimum component's geometry while maintaining its structural integrity in withstanding the given loads, both static and fatigue. Since the MLG fitting is one of the Principal Structural Elements (PSEs) of the aircraft, the failure of this component can lead into a catastrophic accident. Hence, a damage tolerance analysis is essential to be conducted in order to determine the service life and the inspection interval of the fitting. Here, a combination of the short- and long-range flights for the aircraft was used to construct the load spectrum utilised in fitting's crack growth examination. It is expected that the implementation of both the topology optimisation and the damage tolerance analysis on the fitting could significantly reduce the weight of the fitting while maintaining the compliance to the required static strength and damage tolerance aspects as stated on the Federal Acquisition Regulation (FAR) 23.
\end{abstract}

Keywords: Crack Growth; Fatigue; Fracture Mechanics; Principal Structural Element; Weight Reduction

\section{Introduction}

Aircraft structural components are designed meticulously to achieve the lightest weight possible while still giving a sufficient strength to withstand the loads during its operations. Aircraft is known as an engineering design product that adopts lightweight structures. A lightweight structure is a type of structure that utilises lightweight and high strength materials as well as advances technologies for their design and construction (Zhu et al., 2018).

Topology optimisation of the MLG fitting is targeting in a weight reduction while maintaining its structural strength. Afterwards, the optimised structure will be simulated and compared to the initial configuration to prove its strength and integrity. The simulation is done by utilising the software owned by Altair known as HyperWorks. The pre-processing stage will be conducted using HyperMesh. The OptiStruct will be used as the solver for the optimisation process, while the result will be viewed on the HyperView. 
The topology optimised MLG fitting will go through the damage tolerance analysis (DTA) to see its new tolerance compared to the initial design. MLG fitting is one of several Principal Structural Elements (PSE) exists on an aircraft as seen in Fig. 1. Principal structural element, PSE, is an element that contributes significantly to the carrying of flight, ground, or pressurization loads, and whose integrity is essential in maintaining the overall integrity of the airplane (Administration, 2011). This makes the fitting as one of several critical components of the aircraft that the failure of this component will cause a catastrophic event. In addition, there are four fittings that connect the main landing gear to the fuselage structure.

Figure 1: Main Landing Gear Fitting to the Fuselage Structure

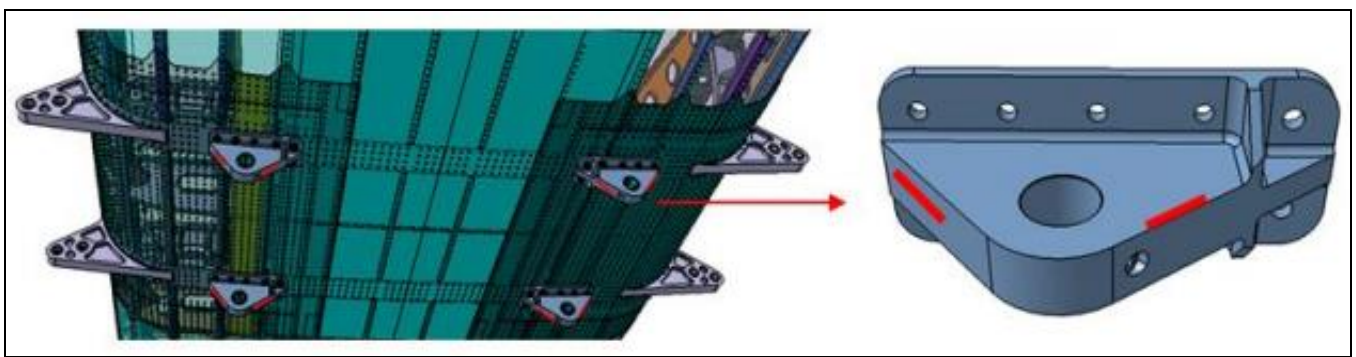

Source: (Filbert, 2019)

As the failure of the MLG fitting is disastrous, thus a damage tolerance evaluation is vital. For a metallic airframe structure, the damage tolerance evaluation must include determination of the probable locations and modes of damage due to fatigue, corrosion, or accidental damage. In addition, an evaluation of the strength, detail design, and fabrication must show that catastrophic failure due to fatigue, corrosion, defects, or damage will be avoided throughout the operational life of the airplane (Administration, 2003). The fitting uses AISI 4340, in which considered as a high-strength low alloy steel material (Wanhill et al., 2010). The physical and the material properties of the original MLG fitting is given in Tab. 1.

Table 1: The Physical and Material Properties of the Original MLG Fitting

\begin{tabular}{l|c}
\hline Properties & Values \\
\hline Volume $\left(\mathrm{mm}^{3}\right)$ & 486,528 \\
Mass $(\mathrm{g})$ & 3,819 \\
Density $\left(\mathrm{g} / \mathrm{mm}^{3}\right)$ & $7.85 \times 10^{-3}$ \\
Young's Modulus $(\mathrm{MPa})$ & 207,000 \\
Yield Strength $(\mathrm{MPa})$ & 1,178 \\
Ultimate Tensile Strength $(\mathrm{MPa})$ & 1,240 \\
Poisson's Ratio & 0.33 \\
Plane Strain Fracture Toughness, $\mathrm{K}_{\mathrm{IC}}\left(\mathrm{MPa}^{\mathrm{N}}{ }^{1 / 2}\right)$ & 69.6 \\
\hline
\end{tabular}

Source: (Autodesk Inventor, 2020; Newman, Jr., 1976)

Damage tolerance is the competence of a structure in maintaining its strength as there are existing defects (i.e. may be present initially due to manufacturing process) up until a corrective action was taken. The defect may exist in the form of an initial crack opening. During the DTA of the MLG fitting, an initial crack is applied. The output of the DTA consists of the fitting's graph of fatigue crack growth rate and residual strength. Both graphs give further estimate of the inspection interval of the fitting, meaning that after certain cycles (or flight hours) an inspection has to be conducted to check the status of the crack size and the remaining strength. 


\subsection{Assumptions}

During the analysis, with a regard of simplification, the assumptions that were used are:

a) The analysis was done only due to Load Case 3 (Filbert, 2019).

b) The reference coordinate axis will follow the body-fixed system as referring to ANSI/AIAA R-0004-1992 Recommended Practice for Atmospheric and Space Flight Vehicle Coordinate Systems (Weiland, 2014).

\section{Topology Optimisation and Damage Tolerance Analysis of MLG Fitting}

In this paper, topology optimisation is a method that is used in optimising the MLG fitting by utilising the OptiStruct software. The software uses the gradient-based optimisation method that will maximise the weight reduction of the fitting while maintaining its structural integrity. The DTA will then being conducted by applying an initial part-through crack at the location where the principal stress under tension is maximum.

\subsection{Topology Optimisation of the MLG Fitting}

The process of optimisation is the work that was done in obtaining the optimum solution with the ultimate end goal that the design objective and constraints are met. Structural optimisation targeted the minimisation of constraints. The reduction on the MLG fitting's weight is desired due to stress and deflection constraints. The weight decrease of the fitting is beneficial in terms of the manufacturing and the operating cost. Lighter weight may lead into less material needed to create the fitting and thus may deduct the manufacturing cost. The general expression of structural optimisation is shown in Eq. 1 (Larsson, 2016), where $x$ is design variable(s) (i.e. MLG fitting), $f(x)$ is the optimisation's objective (i.e. weight reduction), $g(x)$ is the inequality constraints, and $h(x)$ is the equality constraints.

$$
\left\{\begin{array}{l}
\text { Minimize } f(x) \\
\text { Subjected to }\left\{\begin{array}{l}
g(x) \leq 0 \\
h(x)=0
\end{array}\right.
\end{array}\right.
$$

Topology optimisation aims in a uniform material distribution along the MLG fitting as the domain. In other words, the term of topology optimisation can be used to determine information on the optimum number, shape, and size of openings within a user defined structural domain (Tsavdaridis, Kingman, \& Toropov, 2015). This technique allows the modification of the design's topology, for example by adding holes to the fitting. The optimisation constraint in this technique would be the total volume of the fitting and the objective would be to minimise it. Solid Isotropic Material with Penalisation (SIMP) has been developed years ago, targeting in a wider application of structural optimisation. SIMP method (also known as density method) predicts the best material distribution for the given design space due to the applied loads and boundaries, and performance requirements. The fitting is discretised into finite element grids called as isotropic solid microstructures. Bendsøe's establishment in the field of structural optimisation is known as "The Concept of SIMP-type (power-law) Methods” (SIMP Method for Topology Optimization, 2019). Fig. 2 shows an instance of discretisation of each element using the binary value to represent the material density distribution, $\rho$, whether the element should be filled with material ( $\rho_{e}=1$, coloured 
black) or it should be emptied ( $\rho_{e}=0$, coloured white). In the real-life applications, the density for each element is ranging from a certain minimum value, $\rho_{\min }$, to 1 , in which $\rho_{\min }$ is greater than zero. The $\rho_{\min }$ is the minimum allowable relative density value for empty elements. On the contrary, SIMP method may produce ambiguous solutions known as the "grey" area, where as the region resulted from the optimisation gives an unclear information whether the design space should be filled with a material or being emptied. Hence, a penalty factor is introduced, and the value is raised with a power greater than one (Dunning, 2011).

Figure 2: Optimised Material Layout of a Loaded Beam

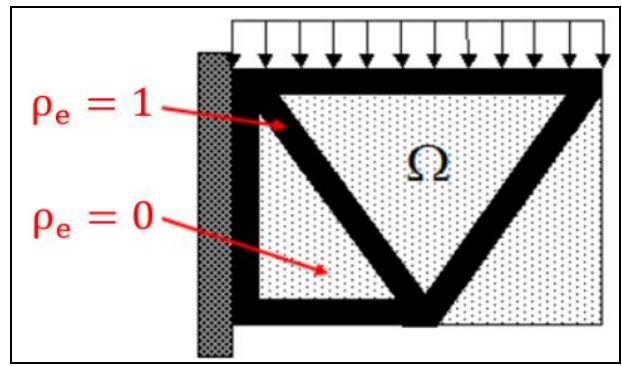

Source: (Dassault Systèmes, 2020)

The topology optimisation by using OptiStruct solved the optimisation procedure once the optimum solution is achieved and converged (Weck \& Kim, 2004). The process targeted in a zero-gradient point as it uses an iterative procedure known as the local approximation method. The applied load that was used during the optimisation process is given in Tab.2, while Fig. 3(a) and 3(b) show the topology optimised MLG fitting obtained from the OptiStruct.

Table 2: The Applied Load for Topology Optimisation of the MLG Fitting

Source: (Filbert, 2019)

\begin{tabular}{c|c|c|c}
\hline Case & X-Axis (N) & Y-Axis (N) & Z-Axis (N) \\
\hline 3 & $-76,520$ & $-15,163$ & $-67,338$
\end{tabular}

Figure 3(a): Optimised MLG Fitting (Element Densities Plot)

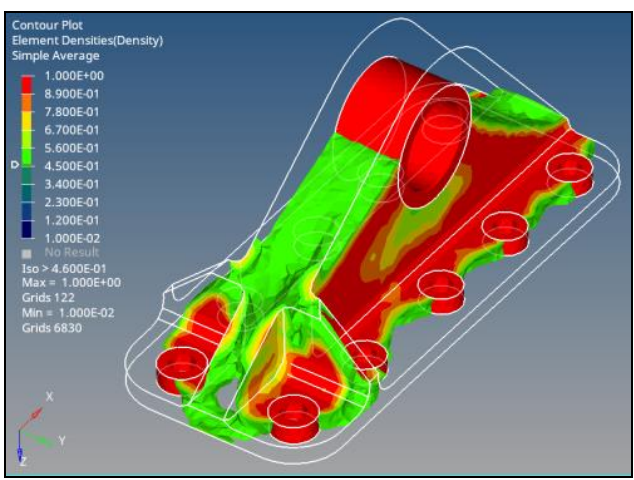

Figure 3(b): Optimised MLG Fitting (Raw)

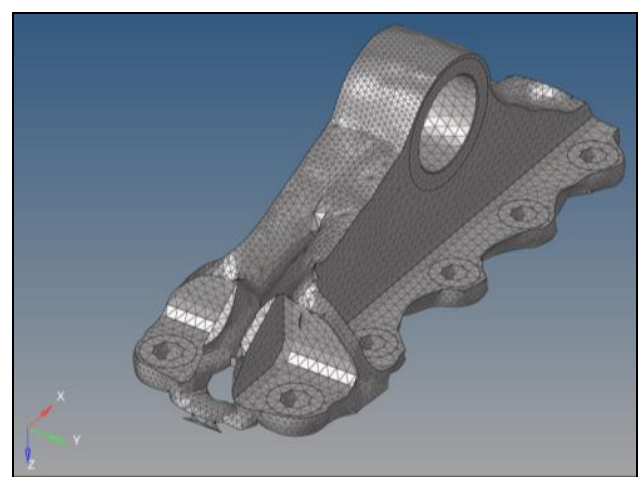

The topology optimised fitting resulted from OptiStruct is still raw and not suitable for manufacturing, hence a smoothing process was done by also considering the damage tolerant. A side-by-side view of the original and smoothed geometry of the fitting is given in Fig. 4(a) and 4(b), respectively. The latter is then being imported to Abaqus for the DTA. In addition, comparing the original and topology optimised MLG fitting, Tab. 3 shows the change of the 
maximum stress and displacement value, and the volume and mass reduction of the MLG fitting.

Figure 4(a): Original MLG Fitting Geometry

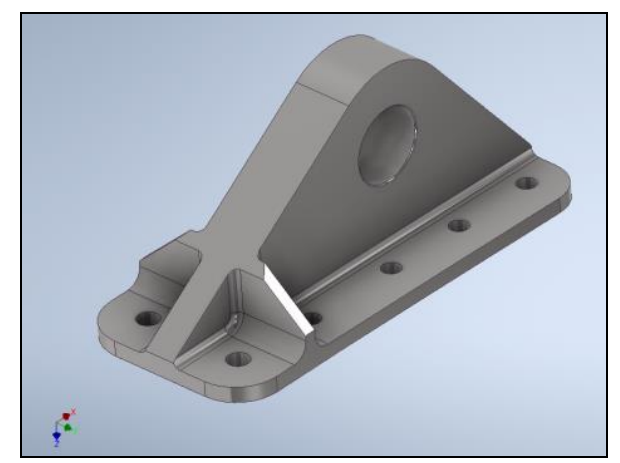

Figure 4(b): Topology Optimised MLG Fitting (Smoothened)

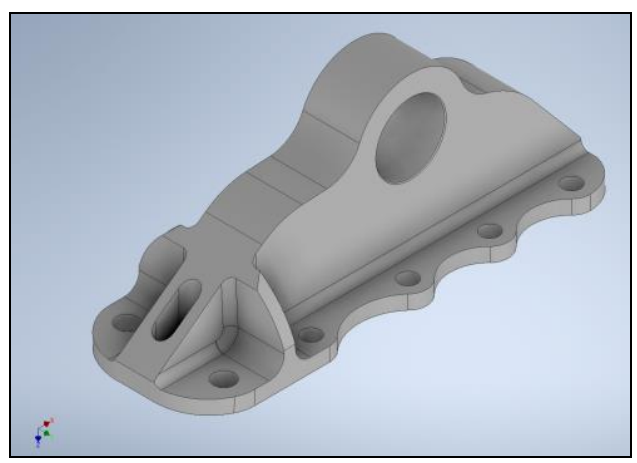

Table 3: Characteristic Alterations between the Original and the Topology Optimised MLG Fitting

\begin{tabular}{l|c|c|c}
\hline Properties & Original & Optimised & Change (\%) \\
\hline Volume $\left(\mathrm{mm}^{3}\right)$ & 486,528 & 388,297 & $\mathbf{- 2 0 . 2}$ \\
Mass $(\mathrm{g})$ & 3,819 & 3,048 & $\mathbf{+ 1 3 . 9}$ \\
Maximum Stress (MPa) & 548.0 & 624.4 & $\mathbf{+ 0 . 7}$ \\
Maximum Displacement (mm) & 0.07387 & 0.07441 & $\mathbf{+}$
\end{tabular}

\subsection{Damage Tolerance Analysis of the MLG Fitting}

Safety requires a structural design that can still withstand an appreciable load under the presence of cracks or failed parts (Broek, 1982). Initial flaws or cracks may exist in the location where the maximum stress concentration is high and it is due to several causes, such as manufacturing, assembling, and service-induced damage. Here, the static loading analysis of the original and optimised MLG fitting was conducted to validate the structural integrity of the smoothened topology optimised fitting. Furthermore, the Stress Intensity Factor, SIF, was obtained from the analysis in Abaqus.

\subsubsection{Cracking Scenario}

As seen in Fig. 5, the stress distribution under the static loading simulation in Abaqus spoils the location of the maximum principal stress, where the corner crack will be applied. Moreover, the size of the initial corner crack given to the fitting and the appearance of the fitting right before the DTA is conducted is given in Fig. 6(a) and 6(b), respectively.

Figure 5: Location of the Maximum Principal Stress 
Figure 6(a): Initial Corner Crack Size

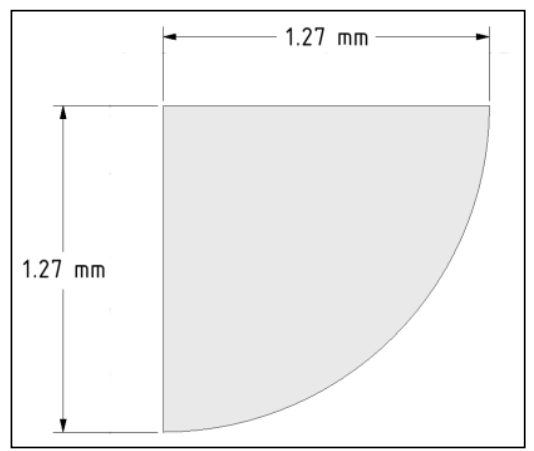

Figure 6(b): Optimised MLG Fitting with an Initial Corner Crack

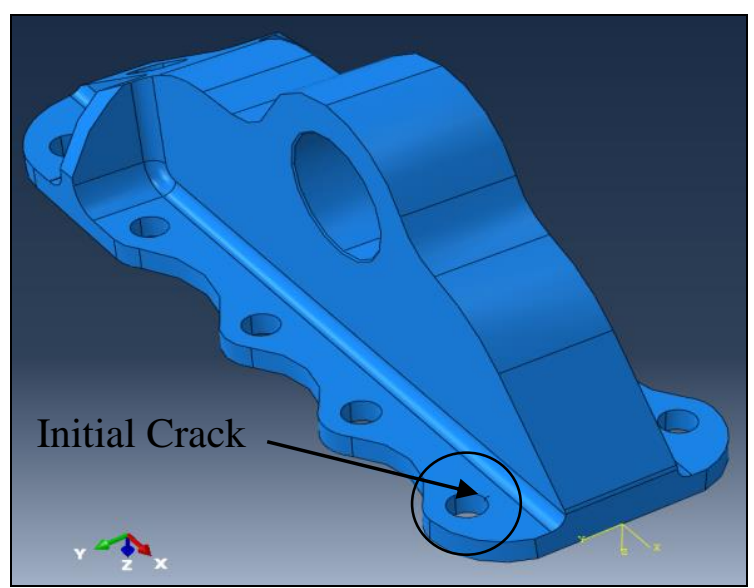

The corner crack or the edge crack will propagate into two directions, however, as the crack's depth goes near the length of the thickness, a critical value of the stress intensity factor is reached. Hence, the MLG fitting is assumed to discover fracture immediately.

\subsubsection{SIF and Dimensionless Shape Factor or Beta-Factor $(\boldsymbol{\beta})$}

The DTA was utilising the Mode I for the stress intensity factor, thus only the value of $K_{I}$ will be used from the result in Abaqus. Analysis on several crack sizes were conducted to be able to plot the alteration of $\beta$ as the crack length rises based on Eq. 2 (Murakami, 2017).

$$
\beta=\frac{K_{I}}{\sigma \sqrt{\pi a}}
$$

The trend line for the change of the Beta-factor as shown in Fig. 7(a) and 7(b) can be pulled out and is being used to construct both the crack growth and the residual strength curves.

Figure 7(a): Trend Line of Beta-Factor (Original)

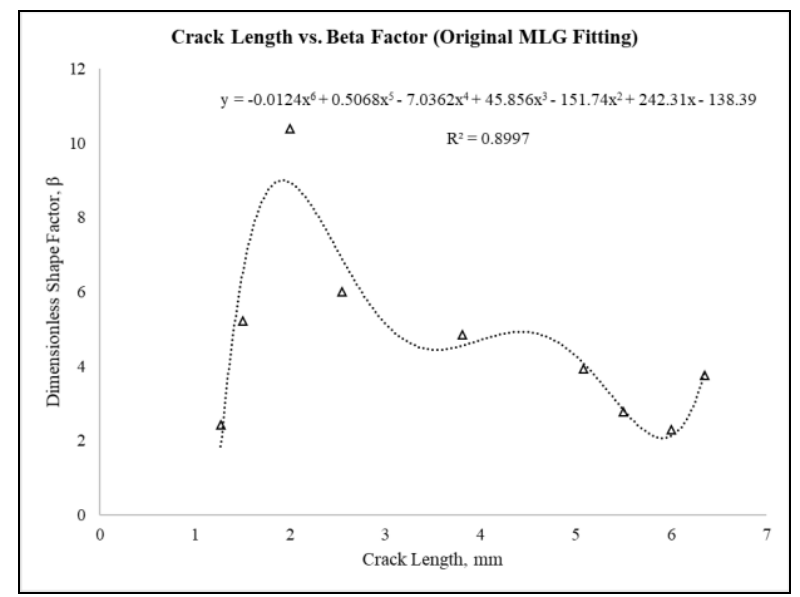

Figure 7(b): Trend Line of Beta-Factor (Optimised)

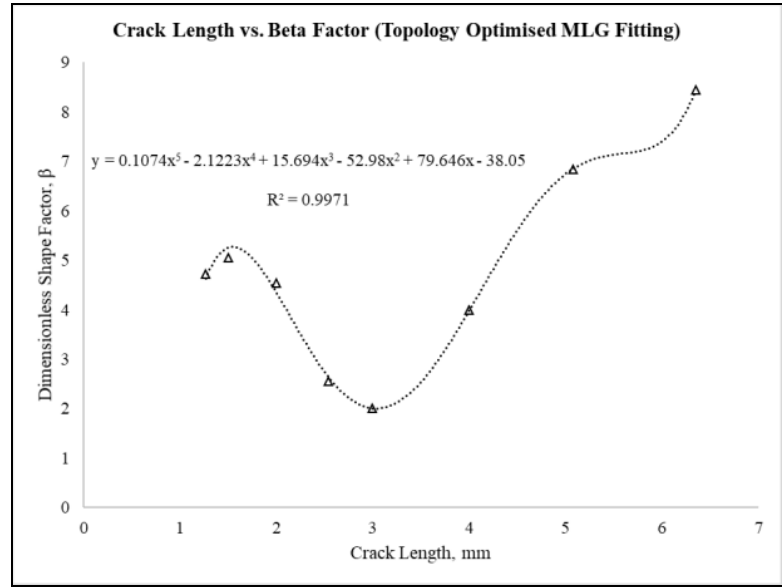

\subsubsection{Crack Growth and Residual Strength Curves}

Determining the number of allowable cycles to occur was done step-by-step. During each cycle, the crack length and the stress intensity factor were monitored. The critical condition will occur once the stress intensity factor in a certain cycle exceeds the fracture toughness of 
the material. In each cycle, the growth of the crack follows the so called as the Paris Equation shown in Eq. 3 (Toribio et al., 2017). The growth in each loading cycle is dependent and directly proportional to the change in the stress intensity factor, $\Delta K$. The terms $C$ and $m$ are both constants and are material properties.

$$
d a / d N=C(\Delta K)^{m}
$$

Cycle-by-cycle, the fitting was analysed using the Constant Amplitude (CA) loading condition as one of the methods to complete the DTA. The approach uses the same load magnitude in each cycle up until the fracture occurred. Thus, the utilisation of Eq. 3 along with the application of CA loading produced the information in Tab. 4 that gives the details about the crack growth and residual strength of both the original and the optimised fitting when it failed.

Table 4: Properties of the Original and Topology Optimised MLG Fitting during Fracture

\begin{tabular}{c|c|c|c|c|c}
\hline Geometry & $\begin{array}{c}\text { Applied Stress } \\
(\mathbf{M P a})\end{array}$ & $\begin{array}{c}\text { Critical Crack } \\
\text { Length }(\mathbf{m m})\end{array}$ & $\begin{array}{c}\text { Residual Strength } \\
(\mathbf{M P a})\end{array}$ & $\begin{array}{c}\text { Stress Intensity Factor } \\
(\mathbf{M P a . m m})\end{array}$ & $\begin{array}{l}\text { Life Estimation } \\
(\mathbf{C y c l e s})\end{array}$ \\
\hline $\begin{array}{c}\text { Original } \\
\text { Topology }\end{array}$ & 45 & 6.83156 & 45.1074 & 2195.71 & $\mathbf{3 3 , 7 4 3}$ \\
Optimised & & 6.68247 & 45.1699 & 2192.67 & $\mathbf{3 4 , 5 9 7}$ \\
\hline \multicolumn{7}{r}{}
\end{tabular}

In addition, the overlaid crack growth and residual strength curves of both the original and the topology optimised fitting is given in Fig. 8(a) and 8(b), accordingly.

Figure 8(a): Crack Growth Rate Curve (Overlaid)

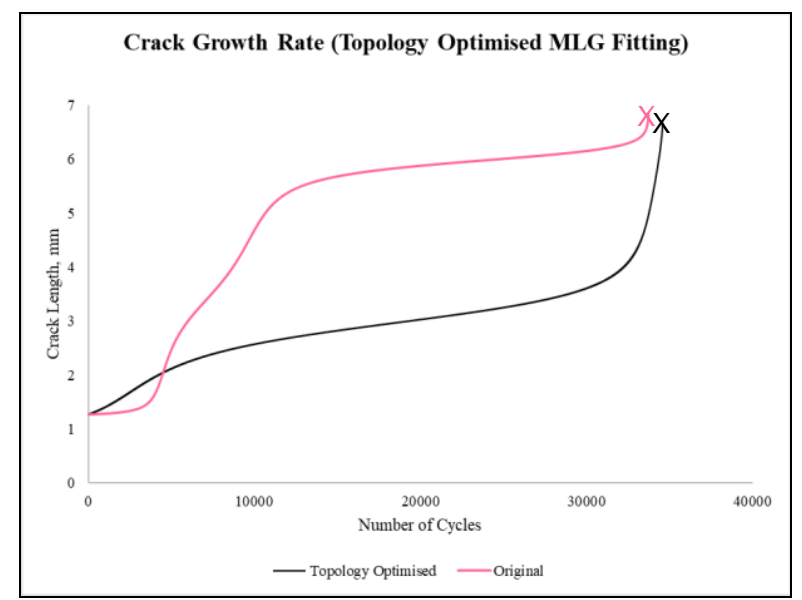

\section{Figure 8(b): Residual Strength Curve (Overlaid)}

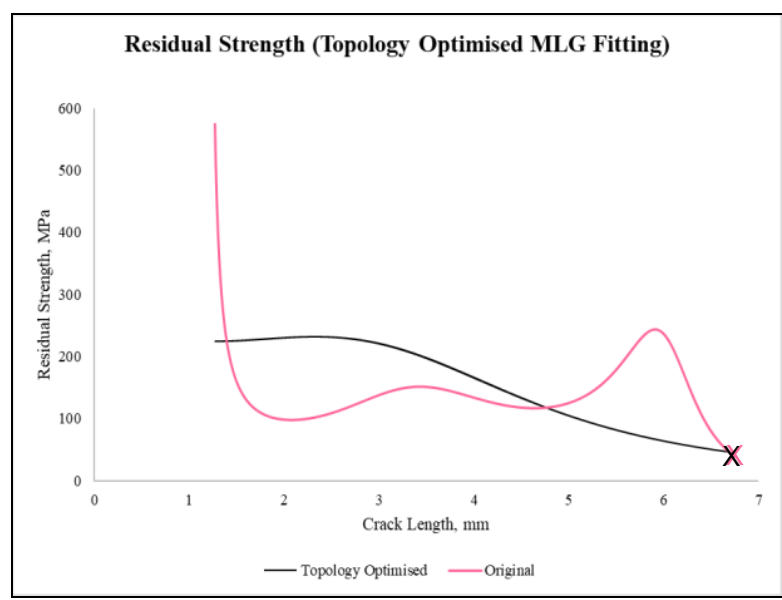

\subsection{Design Review and Inspection Interval Recommendation}

The original geometry of the MLG fitting is analysed and is having the life of 33,743 cycles. On the other hand, the topology optimised fitting can live up to 34,597 cycles by considering only the Mode 1 fracture scenario in enabling the crack to propagate. The $2.53 \%$ longer lifespan is believed due to the desertion of the Mode 2 and Mode 3 fractures that in the future would be a great thing to examine. Furthermore, the life of the topology optimised fitting can be even higher once the applied stress to the structure is lowered as a graph containing the variation of the applied load against the predicted life cycle is given in Fig. 9. It is also supported by the directly proportional relation of the $K$ and $\sigma$ from Eq. 2 . 
Figure 9: Variations of Applied Stress vs. Predicted Cycles (Original \& Optimised)

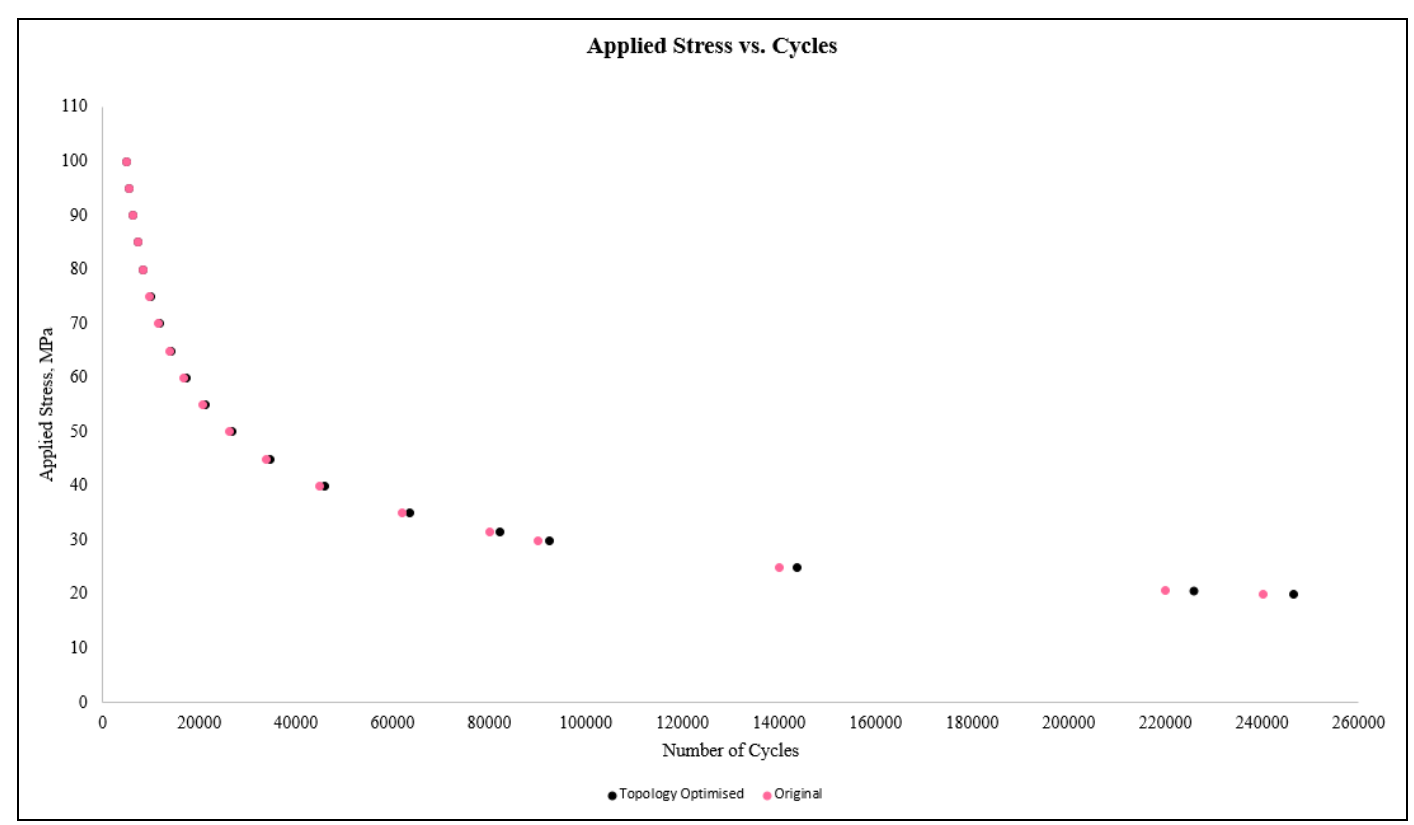

Common crack detection methods in the industry is able to distinguish a crack with a size of four millimetres at the smallest. By considering that, hence following the expression in Eq. 4, the inspection interval for the topology optimised fitting is tabulated as in Tab. 5.

$$
I=\frac{a_{c r i t}-a_{\mathrm{det}}}{3}
$$

Where $I$ is the inspection interval in cycles, $a_{c r i t}$ is the critical crack length at fracture in millimetres, and $a_{\text {det }}$ is the detectable crack length measured by the device in millimetres.

Table 5: Inspection Interval Recommendation for the Topology Optimised MLG Fitting

\begin{tabular}{c|c|c}
\hline $\begin{array}{c}\text { Crack Length } \\
(\mathbf{m m})\end{array}$ & $\begin{array}{c}\text { Crack Growth from Last Inspection } \\
(\mathbf{m m})\end{array}$ & $\begin{array}{c}\text { Number of Cycles before } \\
\text { Upcoming Inspection }\end{array}$ \\
\hline 4.000 & DETACTABLE CRACK SIZE & 32,243 \\
4.894 & 0.894 & 1,443 \\
5.788 & 0.894 & 565 \\
6.682 & 0.894 & CRITICAL CRACK SIZE
\end{tabular}

Tab. 5 shows that the first inspection was persuaded to be done after 32,243 flight cycles, followed by the second inspection with $\mathbf{1 , 4 4 3}$ flight cycles after the first inspection, and the third inspection is recommended to be done 565 flight cycles after the second inspection was conducted. The total number of cycles needed for the optimised component to fail due to the existence of crack subjected to the given stress from the beginning is equal to $\mathbf{3 4 , 5 9 7}$. 


\section{Conclusion}

In conclusion, the process of topology optimisation for the MLG fitting is not only beneficial for the weight reduction of the component, but it is also possible to maintain its structural integrity, in terms of being damage tolerant. The topology optimised fitting is proven to have a total weight decrement of $20.2 \%$ while maintaining its static strength due to the applied load to be within the elastic region or the yield strength of the material. Furthermore, the lifespan of the topology optimised fitting is also have proven to be comparable with the lifespan of the original one, by only considering the Mode 1 fracture scenario, with a $2.53 \%$ margin. With a slightly longer lifetime, the suggested interval for inspection is following the analysis' result that is served in Tab. 5.

\section{Acknowledgment}

I want to express all of my gratitude and thankfulness towards Allah SWT for the helps and guidance in completing this work. Both of my parents, Mun'im Mahalik and Nike Farida Poespitarini that always by my side and keeps believing in myself. Professor Tatacipta Dirgantara, Dr. Annisa Jusuf, and Dr. Leonardo Gunawan as my supervisors back in Institut Teknologi Bandung that always gave me great inputs and amazing discussions regarding the completion of this paper. And lastly, to Virginia Mentari and all of my colleagues back in Indonesia for the amazing love and support that kept me from quitting.

\section{References}

[1] Administration, F. A. (2003). Federal Aviation Regulation. Washington, D.C.: U.S. Department of Transportation.

[2] Administration, F. A. (2011). Advisory Circular No. 25.571-1D: Damage Tolerance and Fatigue Evaluation of Structure. Washington, D.C.: U.S. Department of Transportation.

[3] Broek, D. (1982). Elementary engineering fracture mechanics. Boston: Nijhoff.

[4] Dunning, P. D. (2011, May). Introducing Loading Uncertainty in Level Set-Based Structural Topology Optimisation. Bath, Somerset, United Kingdom.

[5] Filbert. (2019). Topology Optimization of N219 Aircraft Main Landing Gear Fitting Using SIMP Method. Institut Teknologi Bandung, Department of Aerospace Enginnering. Bandung: Faculty of Mechanical and Aerospace Engineering.

[6] Larsson, R. (2016). Methodology for Topology and Shape Optimization: Application to a Rear Lower Control Arm. Göteborg, Sweden.

[7] Murakami, Y. (2017). Theory of Elasticity and Stress Concentration. Chichester: West Sussex, United Kingdom.

[8] Newman, Jr., J. C. (1976). Predicting Failure of Specimens with Either Surface Cracks or Corner Cracks at Holes. Washington, D.C.: National Aeronautics and Space Administration.

[9] SIMP Method for Topology Optimization. (2019). Retrieved November 29, 2019, from SOLIDWORKS Help Web Site: http://help.solidworks.com/2019/english/SolidWorks/cworks/c_simp_method_topology. htm 
[10] Toribio, J., Matos, J.-C., \& González, B. (2017). Paris Law-Based Approach to Fatigue Crack Growth in Notched Plates under Tension Loading. Procedia Structural Integrity, 1299-1303. doi:10.1016/j.prostr.2017.07.115

[11] Tsavdaridis, K. D., Kingman, J. J., \& Toropov, V. V. (2015). Application of structural topology optimisation to perforated steel beams. Computers \& Structures, 108-123. doi:10.1016/j.compstruc.2015.05.004

[12] Wanhill, R. J., Byrnes, R. T., \& Smith, C. L. (2010). Stress corrosion cracking in aerospace vehicles. Amsterdam: National Aerospace Laboratory NLR.

[13] Weck, O. d., \& Kim, I. Y. (2004, January 23). Design Optimization. Cambridge, Massachusetts, United States of America.

[14] Weiland, C. (2014). Coordinate Systems. In Aerodynamic Data of Space Vehicles (pp. 333-334). Springer, Berlin, Heidelberg.

[15] Zhu, L., Li, N., \& Childs, P. (2018). Light-weighting in aerospace component and system design. Propulsion and Power Research, 103-119. doi:10.1016/j.jppr.2018.04.001 